Original article

\title{
The effect of peer education on quality of life in heart failure patients: A randomized clinical trial
}

\author{
Farshad Heidari Beni', Seyyedeh Roghayeh Ehsani², Amir Tabatabaee ${ }^{3}$, Esmaeil Mohammadnejad $^{4 *}$
}

\author{
(Received: 29 Oct 2016; Accepted: 11 Dec 2016)
}

\begin{abstract}
Background and Purpose: Heart failure (HF) is a chronic, debilitating disease, and physical limitations and progressive symptoms of which associated with this condition can reduce quality of life (QOL). Patient education regarding heart HF is one of the main components of self-care. In this respect, peer education is significant in improving health and creating a learning environment. This study was performed to determine the effect of peer education on QOL in HF patients.

Methods: In this quasi-experimental study performed during 2016, 60 HF patients affiliated to Tehran University of Medical Sciences were randomly divided into intervention and control groups $(n=30)$. After preparing the peer group, four peer-to-peer training sessions were conducted during one month. The Persian version of Ferrans and Powers' Quality Of Life Questionnaire was used for data collection, which was completed by both groups before, just, and one month after the intervention. SPSS version 21 was employed for data analysis. Results: No significant difference was observed between the two groups in overall QOL score and its domains before the intervention $(P=0.31)$. The mean scores of QOL immediately after the intervention were $126.2 \pm 6.7$ and $163.1 \pm 1.6$ in the case and control groups, respectively. The

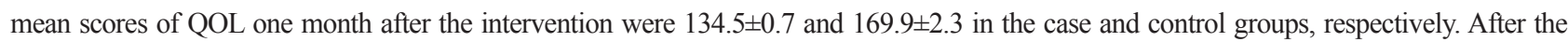
intervention, paired t-test showed that peer education had a significant relationship with QOL of the HF patients $(P<0001)$.

Conclusion: Based on this study, peer education intervention can be a beneficial educative-supportive approach, which might enhance QOL in HF patients.
\end{abstract}

Keywords: Heart failure patients, Peer education, Quality of life

\section{Introduction}

Heart failure (HF) is considered as one of the most important chronic, progressive, and debilitating diseases that despite medical advances is still a major problem in healthcare systems (1). According to the World Health Organization, cardiovascular disease is going to be the leading cause of mortality around the world by 2020 . In Iran, chronic diseases are responsible for about $70 \%$ of deaths and cardiovascular diseases comprise $42 \%$ of this rate (2).

The prevalence of cardiovascular diseases is on a growing trend due to increased life expectancy of these patients and advances in disease treatment. Its prevalence rate is estimated to range between $0.4 \%$ and $2 \%$ among the general population and $0.16 \%$ and $2.3 \%$ among the population aged over 75 years (3). About 5.8 million Americans suffer from HF and 670,000 new cases are identified annually. This

\footnotetext{
${ }^{1}$ School of Nursing and Midwifery, Iran University of Medical Sciences, Tehran, Iran

${ }^{2}$ Department of Nursing, Imam Khomeini Hospital Complex, Tehran University of Medical Sciences, Tehran, Iran

${ }^{3}$ Department of Nursing, Quchan Branch, Islamic Azad University, Quchan, Iran

4,* Corresponding author: Department of Nursing, Student Research Committee, Shahid Beheshti University of Medical Sciences, Tehran, Iran. Email: asreno1358@yahoo.com
} 
condition has affected $1-2 \%$ of the $50-59$ year-old population and more than $10 \%$ of people aged over 70 years. HF is the most common cause of hospital admission in people aged over 50 years and the second leading cause of physician visit in the US (4).

There is a mutual relationship between HF and quality of life (QOL), such that its physical symptoms have a direct impact on all aspects of QOL. Over the past 20 years, efforts have increased to evaluate and improve QOL of chronic disease patients (5). QOL represents a measure of physical and social activity and mental health and is considered as one of the important components of health (6). HF affects all aspects of patient's activities, and subsequently, their QOL to varying degrees (3). The first cause of death in Iran, with an approximate rate of 150,000 per a year, is related to cardiovascular diseases that accounted for a total of $25-40 \%$ of deaths (7). HF is a chronic and debilitating disease whose physical limitations and progressive symptoms result in decreased QOL. Considering the importance of improving QOL in the health care systems, evaluating QOL in patients with HF can help nurses provide a more standard care (8).

Education of HF patients is one of the main components of self-care and includes information about the signs and symptoms of the disease, lifestyle changes, and adherence to the treatment plan. Patients with HF need education and support regarding their medication regimen, drug consumption, diet, and exercise and should take appropriate measures when symptoms present (9). One type of patient education is peer education, which can promote health and create a learning environment (10). Peer education can help increase motivation to receive treatment and reduce regression to high-risk behaviors (11).

Contradictory findings were reported on the impact of peer education (12). Due to the shortage of nurses in hospitals in Iran, they seem to lack sufficient time for communication with patients; thus, training peer groups can resolve this shortcoming. Peer groups are better able to communicate with their counterparts and encourage them to adopt healthy behaviors. Furthermore, this type of training is associated with some economic and social benefits, as well as health improvement. Therefore, this study was performed to determine the effect of peer education on QOL in HF patients.

\section{Materials and Methods}

This quasi-experimental study was conducted to investigate the effect of peer education on QOL of HF patients in Iran, 2016. The study population included all patients with HF that were admitted to the Cardiology Ward. Sixty HF patients (calculated using the following formula) who met the inclusion criteria were selected randomly. For random sampling we used baseline measurements in the ward.

$$
n=\frac{N z^{2} a / 2 P(1-p)}{(N-1) d^{2}+z^{2} a / 2 P(1-p)}
$$

The inclusion criteria were not having any experience of participation in training programs on HF during the past six months, willingness to participate in the study, literacy, having the ability to speak Persian, not having any cognitive difficulties or physical disabilities, availability of medical records of the patient, not having education associated with medical field, and the possibility of establishing a direct telephone contact with the patient or his/her next of kin.

Data collection tools included personal characteristics (age, gender, marital status, educational level, job, duration of disease, having other diseases such as diabetes and hypertension, and educational experience) and QOL questionnaire. This questionnaire was designed in 1999 by Ferrans and Powers. In this questionnaire, the items were rated using a 6-point Likert scale including very low (1), low (2), somewhat (3), medium (4), high (5), and very high (6) (11). Given that the number of questions was 35 , the highest and lowest possible scores were 210 and 35 , respectively.

Three one-hour sessions for all the patients were held by the researcher. In order to ensure the integration of peer groups' education and their readiness, the researcher monitored the process 
of their education using a self-made checklist, consisting of 15 items. In the initial stage, the patients were introduced to peer groups and were presented the objectives and regulations. The educational content was associated with HF. To control educational behaviors, the use of proper audiovisual aids and discussion between learners was checked. Evaluation was made using a summary of the group's questions and answers. The checklist was answered as "Yes" or "No" and to each of the items of Yes and No scores 1 and 0 were allocated, respectively. The total score of the checklist was 15 , and if the peer scores were less than 13, they were removed from peer education groups. The researcher examined all the situations and if the peer complied with the trained content and attained a higher score than 13, s/he was given permission to teach their peer group. Finally, of all the materials introduced in the class, a booklet containing the concepts and benefits of peer-led education and educational needs of $\mathrm{HF}$ patients, including the definition of HF, mechanisms and causes of symptoms, risk factors, the general principles of treatment, drug therapy, non-pharmacological management, body weight management, and diet, was prepared and provided to the peer groups. The patients were divided into several groups of 6-8 to facilitate the education, and then eight patients were randomly referred to a peer educator for special education. The content of the educational sessions was similar for all the patients. The data was analyzed by SPSS version 21 using descriptive and inferential statistical tests.

For the ethical considerations, this study was approved by Student Research Committee of Shahid Beheshti University of Medical Sciences in Tehran (SBUMS). After receiving permission from the Deputy of Research of SBUMS, informed consent was obtained from all the patients and they were assured of the confidentiality of the data. The patients were granted the permission to withdraw from the study at any time.

\section{Results}

Most participants in the intervention (86.7\%) and control (93.3\%) groups were married, $80 \%$ and $83.3 \%$ of the intervention and control groups, respectively, were male. The mean ages of the intervention and control groups were 51.65 \pm 8.3 and $49.23 \pm 6.04$ years, respectively, and the mean durations of the disease in the intervention and control groups were $2.1 \pm 3.1$ and $2.9 \pm 2.8$ years, respectively. The mean scores of QOL in chronic heart failure $(\mathrm{CHF})$ patients before the intervention

Table1. Quality of life in the heart failure patients before the intervention

\begin{tabular}{lcccc}
\hline Areas & Intervention & Control & $\boldsymbol{P}$ & $\mathbf{t}$ \\
\hline Physical dimension & $34.5 \pm 9.1$ & $32.2 \pm 2.5$ & 0.09 & 2.32 \\
Psychological dimension & $29.5 \pm 0.3$ & $31.3 \pm 3.6$ & 1.9 & 1.62 \\
Economic dimension & $33.5 \pm 8.2$ & $33.5 \pm 5.4$ & 0.41 & 0.98 \\
Family dimension & $35.2 \pm 9.1$ & $32.6 \pm 9.4$ & 0.29 & 1.72 \\
Mean quality of life & $123.7 \pm 6.8$ & $128.5 \pm 0.5$ & 0.31 & 1.09 \\
\hline
\end{tabular}

were $128.5 \pm 0.5$ and $123.7 \pm 6.8$ in the control and intervention groups, respectively (Table 1).

Comparison of QOL scores just and one month after the intervention in both groups showed that QOL score increased in both groups and a significant difference was observed in the intervention group (Tables 2 and 3).

Table 2. Quality of life in heart failure patients just after the intervention

\begin{tabular}{lcccc}
\hline Areas & Intervention & Control & $\boldsymbol{P}$ & $\mathbf{t}$ \\
\hline Physical dimension & $42.4 \pm 6.3$ & $31.3 \pm 5.9$ & 0.001 & -1.8 \\
Psychological dimension & $39.3 \pm 0.4$ & $28.3 \pm 0.02$ & 0.04 & 0.18 \\
Economic dimension & $41.2 \pm 4.03$ & $35.1 \pm 2.8$ & 0.008 & -4.07 \\
Family dimension & $40.2 \pm 0.9$ & $31.5 \pm 2.5$ & 0.17 & 5.7 \\
Mean quality of life & $163.1 \pm 1.6$ & $126.2 \pm 6.7$ & 0.31 & 2.14 \\
\hline
\end{tabular}

Table 3. Quality of life in heart failure patients one month after the intervention

\begin{tabular}{lcccc}
\hline Areas & Intervention & Control & $\boldsymbol{P}$ & $\mathbf{t}$ \\
\hline Physical dimension & $41.2 \pm 6.2$ & $32.3 \pm 1.9$ & 0.001 & 10.42 \\
Psychological dimension & $38.9 \pm 1.8$ & $33.9 \pm 0.6$ & 0.001 & 9.36 \\
Economic dimension & $42.1 \pm 3.1$ & $32.8 \pm 6.9$ & 0.001 & 10.03 \\
Family dimension & $46.2 \pm 0.9$ & $35.3 \pm 3.4$ & 0.001 & 6.8 \\
Mean quality of life & $169.8 \pm 2.3$ & $134.5 \pm 0.7$ & 0.001 & 9.27 \\
\hline
\end{tabular}




\section{Discussion}

The purpose of this study was to investigate the effect of peer education on QOL in HF patients. The results of this study showed that the total mean scores of QOL before, just, and one month after the intervention were significantly different. Statistical analysis showed that peer education had a significant impact on improving QOL of HF patients.

The results of Borzu et al. (2014) were consistent with ours. In that study, QOL score before the intervention was not significantly different between the peer and individual groups. Just after the intervention, mean QOL score in both the peer and individual groups was significantly different. Their results showed that both educational methods enhanced QOL, but the longterm effect of peer education was significant (11). The results of Baghaei et al. (2015), similar to our findings, showed that after the intervention, patients' QOL was improved. In physical, emotional, mental, social, and economic dimensions of QOL, a significant difference was observed between the two groups (13).

Study of Fathani et al. (2015) entitled as "the impact of educational intervention on improving the QOL of family caregivers of heart failure patients" showed that the intervention improved the patients' QOL. Any intervention, especially peer intervention, can have a positive impact on the QOL of HF patients. In the peer-led education, a simple and reliable learning environment is created and patients can benefit from the experiences of their peers to improve disease symptoms (14). Abedian et al. (2011) conducted a study to evaluate the impact of peer-led education on healthy behaviors of girls with dysmenorrhea in Kermanshah, Iran. That study showed that knowledge about the physiology of menstruation in the peer group was higher than other groups (15).

Zendehtalab (2013) conducted a study to compare the two methods of individual and group education on QOL in patients with multiple sclerosis by using the Quality of Life questionnaire (SF-36). That study presented that the mean QOL score of the individual learning group had a significant increase compared to the group education (16). A study performed by Khoshraftar Roudi et al. (2015) entitled as "the effect of peer education on the mental aspect of the quality of life of elderly patients with hypertension" showed that the mean score for the psychological aspect of QOL was not significantly different between the two groups before intervention, but after the intervention, the difference in mean scores was significant in this regard (17). The results indicated that peer education is a continuous approach, in which peers and patients set a long-term friendly relationship and share information with each other. Peer-led education can improve social and mental health problems of patients in addition to their treatment problems, and peers can act as mediators between patients and health care providers $(7,18)$. Considering the fact that $\mathrm{HF}$ as a chronic and disabling condition affects different aspects of QOL in the early stages of the disease and that HF patients lack sufficient knowledge about the treatment regimen, timely diagnosis, and control of disease symptoms, paying attention to the peer-led education is of utmost importance.

\section{Conclusion}

The results of this study showed that peer education increased the mean QOL score just and one month after the intervention. Therefore, the use of peer experiences as an easy, cost-effective, and evidencebased method of education is recommended for CHF patients and those with other chronic diseases.

\section{Conflicts of interest}

None declared.

\section{Authors' contributions}

All authors contributed equally to the writing of the scientific proposal, data collection, and manuscript drafting. The final manuscript was reviewed and approved by all the authors.

\section{Acknowledgements}

This study was extracted from a research project, 
approved by Student Research Committee of Shahid Beheshti University of Medical Sciences in Tehran (SBUMS; approval code: 46367/S/2015). The authors would like to thank all the heart failure patients for participating in this study.

\section{References}

1. Borhani F, Khoshab H, Abbaszadeh A, Rashidinejad H, Mohammadi E. Study of the effect of partnership care model on the quality of life in patients with heart failure. J Crit Care Nurs 2012; 5(1):43-8.

2. Abootalebi G, Vosooghi N, Mohammad Nejad E, Namadi M, Akbari Kaji M. Study of the self-care agency in patients with heart failure. J Crit Care Nurs 2012; 4(4):203-8.

3. Zakerimoghadam M, Sadeghi-Akbari A, Cheraghi M, Kazemnejad A. The effect of education based on illness perception on self-care behaviors of patients with heart failure. Cardiovasc Nurs J 2015, 4(1):22-30 (Persian).

4. McMurray JJ, Adamopoulos S, Anker SD, Auricchio A, Bohm M, Dickstein K, et al. ESC guidelines for the diagnosis and treatment of acute and chronic heart failure 2012: The Task Force for the Diagnosis and Treatment of Acute and Chronic Heart Failure 2012 of the European Society of Cardiology. Developed in collaboration with the Heart Failure Association (HFA) of the ESC. Eur Heart J 2012; 33(14):1787-847.

5. Sadeghi Sherme M, Razmjooei N, Ebadi A, Najafi Mehri S, Asadi-Lari M, Bozorgzad P. Effect of applying continuous care model on quality of life of patients after coronary artery bypass graft. J Crit Care Nurs 2009; 2(1):1-6.

6. Moghimian M, Kashani F, Cheraghi MA, Mohammadnejad E. Quality of life and related factors among people with spinal cord injuries in Tehran, Iran. Arch Trauma Res 2015; 4(3):e19280.

7. Eslami R,Sajadi SA, Farsi Z. Comparing the effect of peer education and orientation tour on the stress of patients candidate for coronary angiography in selected hospital of AJA University of Medical Sciences. J Urmia Nurs Midwifery Facul 2015; 12(12):1119-27 (Persian).

8. Yousefi P, Sabzevari S, Mohammadalizadeh S, Haghdoost AA. Study of quality of life in heart failure hospitalized patients in Kerman medical university hospital in 2008.
Iran J Nurs Res 2011; 6(21):59-67.

9. Shojaee A, Nehrir B, Naderi N, Zareiyan A. Effect of patient education and telephone follow up by the nurse on the level of hope in patients suffering from heart failure. $\mathrm{J}$ Nurs Educ 2013; 2(3):16-26 (Persian).

10. Vedel I, Khanassov V. Transitional care for patients with congestive heart failure: a systematic review and metaanalysis. Ann Fam Med 2015; 13(6):562-71.

11. Borzou R, Bayat Z, Salvati M, Homayounfar S. A comparison of individual and peer educational methods on quality of life in patients with heart failure. Iran J Med Educ 2014; 14(9):767-76 (Persian).

12. Khoshraftar Roudi E, Behnam Voshani H, Emami Zeydi A, Askari Hoseini Z, Movahedifar M, Emami Moghadam Z. Comparison of the effects of healthy lifestyle education program implemented by peers and community health nurses on the quality of life of elderly patients with hypertension. Evid Based Care 2016; 5(4):51-60.

13. Baghaei R, Mashallahi A, Khalkhali H. The effect of applying continuous care model on the quality of life in patients with heart failure. J Urmia Nurs Midwifery Facul 2015; 13(8):666-75 (Persian).

14. Fathani M, Afzal Aghaee M, Tadayonfar M. Evaluation of the effect of designated educational intervention on the improvement of quality of life in caregivers of patients with chronic heart failure. J Babol Univ Med Sci 2016; 18(5):20-5.

15. Abedian Z, Kabirian M, Mazlom SR, Mahram B. The effects of peer education on health behaviors in girls with dysmenorrhea. J Am Sci 2011; 7(1):431-8.

16. Zendehtalab H. Comparing the effect of face to face and group discussion teaching methods on quality of life improvement among patients suffering from multiple sclerosis. Iran J Med Educ 2013; 13(9):711-20 (Persian).

17. Khoshraftar RE, Ildarabadi E, Behnam VH, Emami MZ. The effect of peer education on the mental aspect of quality of life of elderly patients with hypertension. J North Khorasan Univ Med Sci 2016; 7(3):585-95 (Persian).

18. Fisher EB, Boothroyd RI, Coufal MM, Baumann LC, Mbanya JC, Rotheram-Borus MJ, et al. Peer support for self-management of diabetes improved outcomes in international settings. Health Aff (Millwood) 2012; 31(1):130-9. 